\title{
Evaluación de las reservas de oro (Au) en las terrazas aluviales del sector San Vicente
}

Evaluation of the gold $(\mathrm{Au})$ reserves in the alluvial terraces of the San

Vicente sector

Marco Antonio Mejía Flores. ${ }^{1}$, Jenny Priscila Granja Carrera. ${ }^{2}$ \& Faustino Ampam

Tsenkush Chamik. ${ }^{3}$

\begin{abstract}
.
DOI: https://doi.org/10.33262/concienciadigital.v4i3.1766

Introduction: In the Amazon region of Ecuador, there are alluvial gold deposits that are extracted by local communities. under the modality of artisanal mining permit, granted by the Ecuadorian State; as is the case of the San Vicente sector, Patuca parish, in Morona Santiago. The extraction is carried out empirically without a prior technical study. For this reason, the field work includes the prospecting of an area of $41.7 \mathrm{ha}$, a model was made determining with the areas with positive mineralization to be used and finally a method of exploitation is proposed. Objective. Carry out the evaluation of the gold ( $\mathrm{Au})$ reserves in the eluvial terraces of the San Vicente Sector, through a technical analysis in order to adopt exploitation systems according to the geological and mineralogical characteristics and the quantity and quality of the mineral existing in the deposit. eluvial. Methodology, consisted of sampling, washing and concentrating of eluvial materials; with a pan and the $10 \mathrm{~kg}$ concentrated material, to detect the presence of minerals such as gold $(\mathrm{Au})$; according to the color chart procedure. Results: - In the alluvial terrace, the volume calculation is established by the method of profiles of high concentration mineralized strata in $159,461.98 \mathrm{~m} 3$ with a content of $0.56 \mathrm{gr} / \mathrm{m} 3$ and reserves of $89,298.70$ grams of gold, medium concentration 100,486, $86 \mathrm{~m} 3$ with a content of 0.085 gr / $\mathrm{m} 3$ and reserves of 8,541.38 grams of gold, low concentration $29,657.25 \mathrm{~m} 3$ with a

\footnotetext{
1 Escuela Superior Politécnica de Chimborazo, Carrera de Minas. Macas, Ecuador. marco.mejia@espoch.edu.ec, https://orcid.org/0000-0002-7566-2063

2 Escuela Superior Politécnica de Chimborazo, Carrera de Minas. Macas, Ecuador. jenny.granja@espoch.edu.ec, https://orcid.org/0000-0003-4005-3687

${ }^{3}$ Investigador Independiente. faustino.ksenkush@espoch.edu.ec, https://orcid.org/0000-0003-2812-8789
} 
content of $0.016 \mathrm{gr} / \mathrm{m} 3$ and reserves of 474.51 grams of gold, obtaining total proven reserves of 98,314 , 59 grams of gold. Conclution. • Strip start-up and hydraulic start-up have a lower operating cost than strip and mechanical start-up, but with greater investment in mitigation and environmental control works.

Keywords: Eluvial terrace; Mineralized strata, Gold reserves, Deposit tenor, Siliceous sand.

\section{Resumen.}

Introducción: En la región amazónica del Ecuador, se tienen yacimientos de oro aluvial que son extraídos por comunidades locales. bajo la modalidad de permiso de minería artesanal, otorgado por el Estado Ecuatoriano; como es el caso del sector San Vicente, parroquia Patuca, en Morona Santiago. La extracción lo realizan de forma empírica sin un estudio técnico previo. Por tal motivo, el trabajo de campo incluye la prospección de un área de 41.7 ha, se hizo un modelo determinando con las áreas con mineralización positiva a utilizar y finalmente se propone un método de explotación. Objetivo. Realizar la evaluación de las reservas de oro (Au) en las terrazas eluviales del Sector San Vicente, mediante un análisis técnico con el fin de adoptar sistemas de explotación acorde a las características geológicas, mineralógicas y la cantidad y calidad del mineral existente en el depósito eluvial. Metodología, consistió en muestreo, lavado y concentrado de materiales eluviales; con una batea y el material concentrado de 10 kilos, para detectar la presencia de minerales como el oro $(\mathrm{Au})$; según el procedimiento de la tabla de colores. Resultados: •En la terraza aluvial se establece el cálculo de volumen por el método de perfiles de estratos mineralizados de alta concentración en $159.461,98 \mathrm{~m}^{3}$ con un tenor de $0.56 \mathrm{gr} / \mathrm{m}^{3}$ y reservas de $89.298,70$ gramos de oro, mediana concentración 100.486,86 $\mathrm{m}^{3}$ con un tenor de $0.085 \mathrm{gr} / \mathrm{m}^{3}$ y reservas de 8.541 .38 gramos de oro, baja concentración $29.657 .25 \mathrm{~m}^{3}$ con un tenor de $0.016 \mathrm{gr} / \mathrm{m}^{3}$ y reservas de 474.51 gramos de oro, obteniéndose unas reservas probadas totales de 98.314,59 gramos de oro. Conclusión. -El arranque por franjas y arranque por medios hidráulicos es de menor coste de operación que el de franjas y arranque por medios mecánicos, pero con mayor inversión de obras de mitigación y control ambiental.

Palabras claves: Terraza eluvial; Estratos mineralizados, Reservas de oro, Tenor del yacimiento, Arena silícea.

\section{Introducción.}

El oro "Au”, (proviene del latín Aurum) es el elemento atómico número 79 de la tabla periódica de los elementos; su densidad es $19,32 \mathrm{gr} / \mathrm{cm}^{3}$; su punto de fusión es de $1.063^{\circ} \mathrm{C}$; su dureza es de 2.5 a 3 (escala de Mohs). El oro físicamente es un metal dúctil y maleable; presenta varias tonalidades de color amarillo, según su pureza (cantidad de oro referente a otros minerales asociados); es insoluble en los ácidos ordinarios, pero es soluble en agua 
regia (mezcla de ácidos clorhídrico y nítrico); no se empaña; en lingote se le fracciona, modela y martilla en frío (Pimentel, N. (2010). El oro en la naturaleza se presenta en dos tipos de depósitos en primario y en secundario: Como depósitos primarios tenemos a las vetas masivas de cuarzo con oro nativo (óxidos), vetas de cuarzo que contienen abundante pirita (sulfuros), zonas piríticas con oro en lavas cizalladas sin cuarzo. En forma de depósitos de origen secundario tenemos a los placeres que se encuentran en la inmediata cercanía de la roca originaria conocidos como yacimientos eluviales (oro libre encontrado en las laderas por encima del afloramiento de una veta aurífera es un eluvión); placeres ubicados en lechos o antiguos lechos de ríos compuestos por material sometidos a gran arrastre denominados yacimientos aluviales o fluviales. (Swiecki, 2008); conforme a las propiedades antes descritas el oro secundario se puede transportar fluvialmente y concentrarse gravimétricamente en la naturaleza; razón por la cual se puede emplear la metodología de "tabla de colores"; que consiste en definir como "color" un rango según el tamaño de partículas de oro o platino encontradas en las gravas auríferas, y ordenar en categorías de mayor a menor tamaño como \#1, \#2, \#3, entre otros; para ser registrado en peso (gramos), el oro encontrado en cada muestreo. Mediante la "tabla de colores" se puede determinar el tamaño de oro recuperable, estimados de producción diaria, estimados de perdidas, estimación de piques o pozos, estimación de tenores a diferentes profundidades o en determinación de oro de avance por avance. La "tabla de colores" es particular y única para cada yacimiento estudiado.

En placeres auríferos el método de extracción a cielo abierto es el más fácil y rápido para explotar un yacimiento, pero solamente es aplicable a los depósitos auríferos pocos profundos cubiertos por terrenos blandos. (Swiecki, 2008). La forma tradicional de explotar artesanalmente es mediante uso de bateas (platón), canaletas, cribas, motobombas hidromonitores y dragas de poco calaje y de forma mecanizada mediante el uso de excavadoras, dragalinas, también hidromonitores y dragas de calaje mayor. Según la legislación ecuatoriana en minería artesanal solo se permite el uso de maquinaria con condiciones de capacidad y potencia mínima. También se puede ejecutar la explotación en forma combinada manual y mecanizada al mismo tiempo.

\section{Metodología.}

\section{Descripción topográfica:}

Los permisos de minería artesanal se encuentran situados en forma contigua sobre una franja de depósito aluvial de arena silícea que se extiende de este a oeste; este sitio va desde un relieve tipo montañoso (estribaciones de cordillera del Cutucú) a una llanura sin un desnivel muy marcado, en una fracción de humedal que funciona como filtro para las aguas que drenan hacia el Río Namangoza. La ubicación de la zona de estudio se encuentra indicada en el Figura $\mathrm{N}^{\circ} 1$. 
Figura 1. Ubicación de la zona de estudio

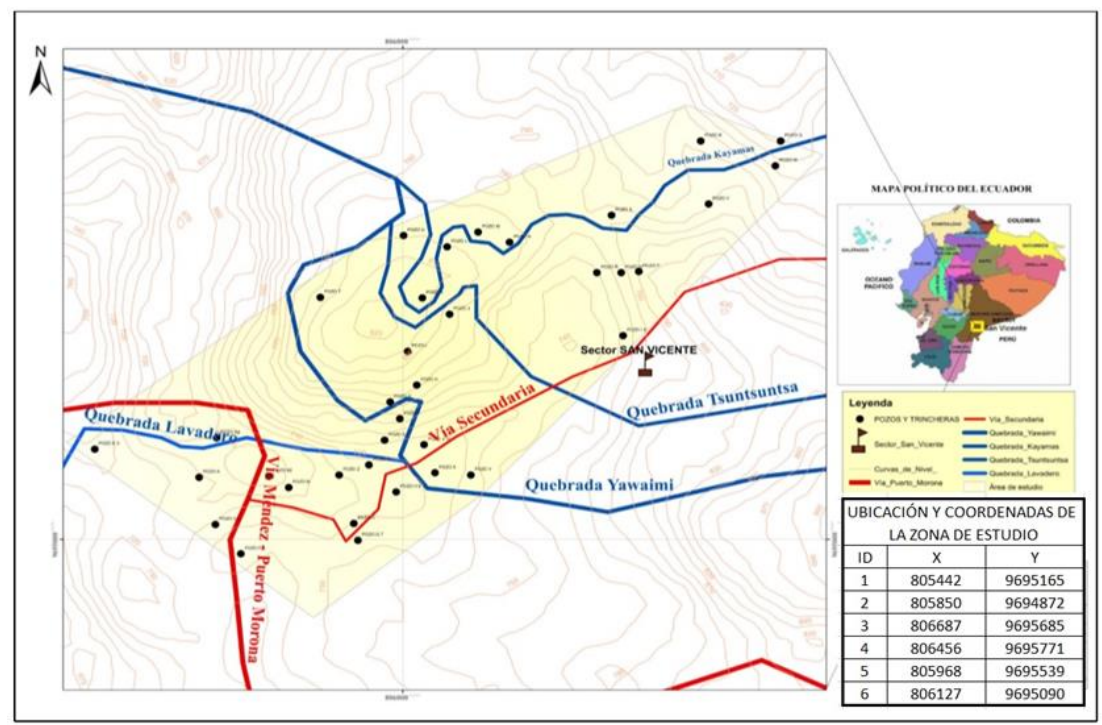

Fuente: Tesis "Evaluación De Las Reservas De Oro (Au) En Las Terrazas Eluviales Del Sector San Vicente" (Tsenkush F, 2016)

Geología Local. Los afloramientos de la zona de estudio están constituidos de arena silícea de color blanco con estratificación cruzada, con paquetes de limos cuarzosos, generalmente de color grisáceo, cubiertas por arcillas de color rojizo. Las areniscas de la Formación Hollín (Cretácico temprano: Albiano - Aptiano) afloran extensamente en la zona subandina, (Baldock W, 1982).

Características técnicas del depósito aluvial. De manera general, de lo obtenido de las observaciones de campo en los afloramientos y calicatas, el yacimiento se encuentra en la base arena silícea entre los 0.5 a $2 \mathrm{~m}$, luego limos cuarzosos con intercalaciones de arcillas con un espesor de 1.5 a 3 metros, continúa con 1.0 a 4.5 metros de suelo residual y finalmente una capa de materia orgánica que va desde los $10 \mathrm{~cm}$ hasta 2,50 m; conforme se indica en el Figura $\mathrm{N}^{\circ} 2$.

Figura 2. Características técnicas del depósito aluvial

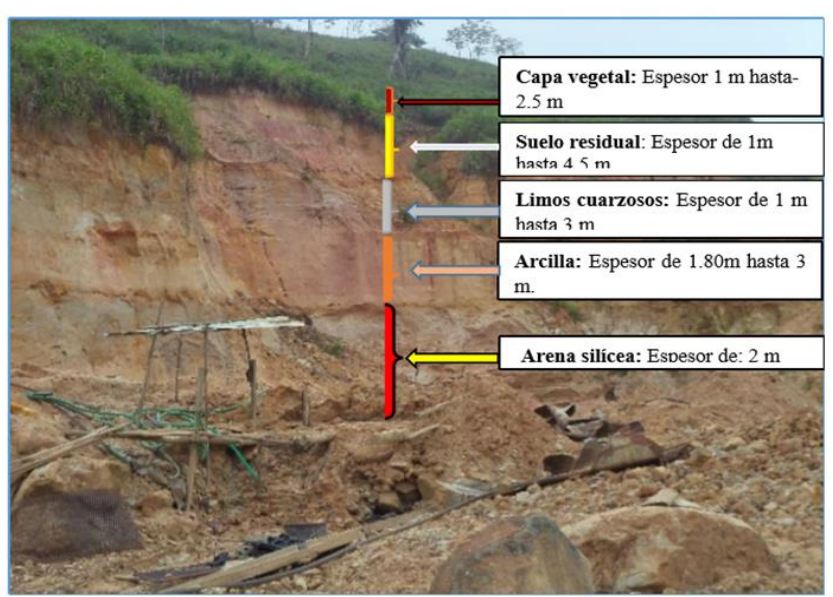

Fuente: Tesis "Evaluación De Las Reservas De Oro (Au) En Las Terrazas Eluviales Del Sector San Vicente" (Tsenkush F, 2016) 
Prospección. Se inició con el trazo de las líneas transversales en los flancos pendientes y en los márgenes derecho e izquierdo de las quebradas: Yawaimi, Kayamas, Tsuntsuntsa y el Lavadero, se continuó con el desbroce de la vegetación que impedía el acceso al lugar, seguido por la limpieza y retiro de la capa vegetal previa la ejecución de calicatas y trincheras, para el efecto se contó con ayuda de mineros artesanales. Las herramientas principales para este tipo de prospección fueron bateas que median $47 \mathrm{~cm}$ de diámetro por $12 \mathrm{~cm}$ de profundidad, recipientes plásticos con capacidad de 21 litros $\left(0.021 \mathrm{~m}^{3}\right)$ con el fin de trabajar por muestra con 10 kilos del material concentrado para aplicar el método indirecto, y también la ayuda de canalones de madera con rifles metálicos de diferentes dimensiones. Se efectuó varias fases en el lavado en batea (disgregación) hasta llegar al mineral concentrado (concentración) mediante los movimientos rotatorios y de desplazamiento longitudinal, lo cual permite concentrar gravimétricamente los minerales pesados en este caso oro libre. Los granos de la batea se van clasificando, los livianos hacia el borde de la batea y los pesados hacia el fondo de esta. La obtención del mineral es cuando la cantidad de arena blanca de cuarzo y sílice fino se reduce a una ligera lámina de arena negra y de trazas, y/o chispas de oro $(\mathrm{Au})$ de color amarillo; este concentrado se coloca en una plancha de metal y se asienta sobre una hornilla a gas, ocupada en el campo con el fin de secar el concentrado para luego separar con imán la arena negra y otros minerales para proceder con facilidad al conteo de oro con la ayuda de dos sets de lupas de 20 X. 30X y 40X. La clasificación se realiza acorde a la metodología de la tabla de colores por cada bateada y al final según los datos obtenidos se pueda estimar y categorizar sitios de baja, mediana y alta concentración de oro (Au). Como se muestra en el Figura $\mathrm{N}^{\circ} 3$.

Figura 3. Método Gravimétrico, lavado y concentrado del oro $(\mathrm{Au})$ en canalón y batea.

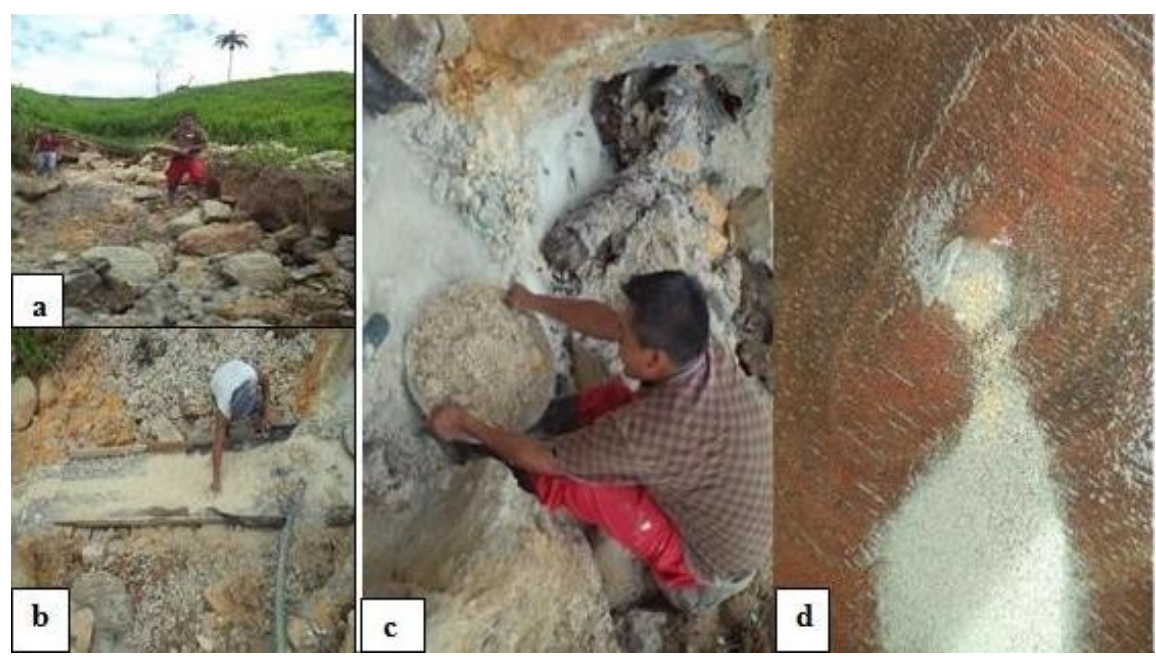

a.- Arranque del material del estrato mineralizado.

b.- Lavado con canalón, disgregación del mineral y control de la operación de concentración en trabajo de prueba.

c.- Lavado en batea, disgregación del mineral y control en la operación de prueba.

d.- Trabajo con el concentrado total obtenido hasta obtener la concentración de arena negra con fragmentos auríferos visibles con el fin de realizar el conteo.

Fuente: Tesis “Evaluación De Las Reservas De Oro (Au) En Las Terrazas Eluviales Del Sector San Vicente" (Tsenkush F, 2016) 
Muestreo en terrazas aluviales mediante pozos y trincheras. Se procedió a cavar pozos de $1.50 \mathrm{~m}$ hasta $3.50 \mathrm{~m}$ de profundidad por lo general en forma manual (Figura $\mathrm{N}^{\circ}$ 4). Hasta llegar o atravesar la capa aurífera (estrato mineralizado) de la cual se toma una muestra de 10 kilogramos para realizar el lavado gravimétrico. Se colocó la muestra en una funda plástica, previamente enumerada en la que consta: nombre del área minera, ubicación geográfica del muestreo, fecha, hora.

En las siguientes figuras se aprecia que el tamaño de los clastos del estrato presenta la siguiente escala: Mayores $30 \mathrm{~cm}=2 \%$; de 20-30 cm=3\%; de10-20 cm= 5\%; menores 1$5 \mathrm{~cm}=20 \%$ y arena silícea $=70 \%$

Figura 4. Recolección de muestras
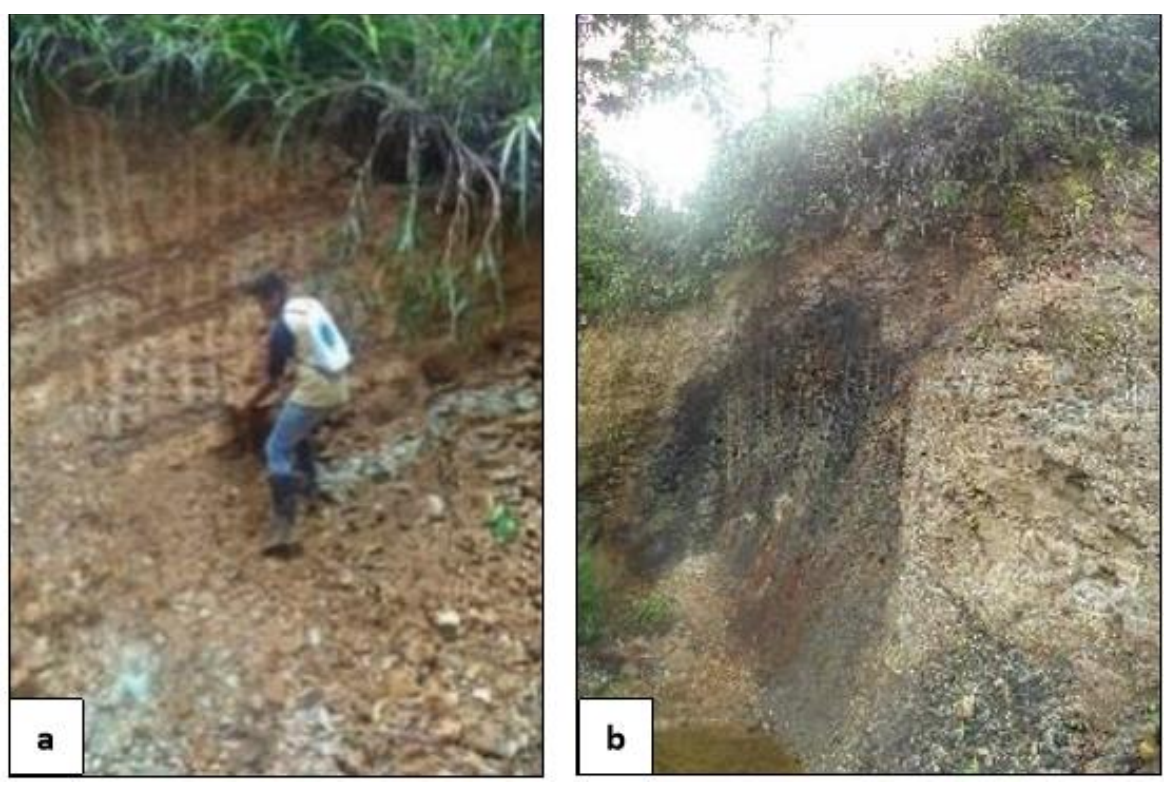

a.- Recolección de la muestra de $10 \mathrm{~kg}$

b.- Calicata de prospección

Fuente: Tesis "Evaluación De Las Reservas De Oro (Au) En Las Terrazas Eluviales Del Sector San Vicente" (Tsenkush F, 2016)

\section{Resultados.}

\section{Determinación de los colores}

Luego del conteo de chispas de las 38 muestras positivas recolectadas en los pozos, calicatas y afloramientos, se observa que su granulometría oscila entre tamaños menores a $1 \mathrm{~mm}$ hasta $0,67 \mathrm{~mm}$ de tal razón se define en tres grupos de colores:

$\begin{array}{lll}\text { Malla } 60 & <0,3 \mathrm{~mm} & \text { Trazas; } \\ \text { Malla } 35 & 0,3-0,5 \mathrm{~mm} & \text { Muy Fino; } \\ \text { Malla } 18 & 0,6-1 \mathrm{~mm} & \text { Fino; }\end{array}$




\section{Determinación del grado de Concentración}

A continuación, se observa las tablas detalladas de acuerdo con el grado de concentración del mineral: alta, media y baja.

Tabla $N^{\circ} 1$ Colores y sitios de alta concentración.

\begin{tabular}{|c|c|c|c|c|c|c|c|c|c|}
\hline \multicolumn{10}{|c|}{$\begin{array}{c}\text { SITIOS DE ALTA CONCENTRACIÓN } \\
\text { COLORES }\end{array}$} \\
\hline $\begin{array}{l}\text { Nombre } \\
\text { pozo }\end{array}$ & $\begin{array}{c}N^{\circ} \text { de } \\
\text { colores } \\
\text { m }^{3}\end{array}$ & $\begin{array}{c}\text { Malla } \\
60 \\
<0,3 \\
\text { mm } \\
\text { Trazas }\end{array}$ & $\begin{array}{l}\text { Peso } \\
\text { mg }\end{array}$ & $\begin{array}{c}\text { Malla } \\
350,3- \\
0,5 \mathrm{~mm} \\
\text { Muy } \\
\text { Fino }\end{array}$ & $\begin{array}{c}\text { peso } \\
\text { mg }\end{array}$ & $\begin{array}{c}\text { Malla } \\
\mathbf{1 8} \\
0,6-1 \\
\mathrm{~mm} \\
\text { Fino }\end{array}$ & $\begin{array}{l}\text { Peso } \\
\text { mg }\end{array}$ & $\begin{array}{c}\text { Peso } \\
\text { total } \\
\mathrm{mg}\end{array}$ & $\begin{array}{l}\text { Potencia } \\
\text { de } \\
\text { sobrecarga } \\
\quad(\mathrm{m})\end{array}$ \\
\hline W & 105 & 6 & 0,072 & 62 & 4,34 & 29 & 10,7 & 15,142 & 15 \\
\hline A & 95 & 9 & 0,108 & 58 & 4,06 & 26 & 9,62 & 13,788 & 12 \\
\hline C3 & 75 & 5 & 0,06 & 43 & 3,01 & 28 & 10,4 & 13,43 & 10 \\
\hline Q & 61 & 7 & 0,084 & 32 & 2,24 & 8 & 2,96 & 5,284 & 10 \\
\hline E5 & 50 & 6 & 0,072 & 28 & 1,96 & 16 & 5,92 & 7,952 & 15 \\
\hline $\mathrm{C}$ & 49 & 5 & 0,06 & 22 & 1,54 & 22 & 8,14 & 9,74 & 10 \\
\hline B & 47 & 7 & 0,084 & 21 & 1,47 & 20 & 7,4 & 8,954 & 3 \\
\hline B 2 & 47 & 6 & 0,072 & 19 & 1,33 & 22 & 8,14 & 9,542 & 4 \\
\hline $\mathrm{R}$ & 45 & 8 & 0,096 & 21 & 1,47 & 16 & 5,92 & 7,486 & 4 \\
\hline D4 & 38 & 2 & 0,024 & 16 & 1,12 & 20 & 7,4 & 8,544 & 3 \\
\hline D5 & 38 & 7 & 0,084 & 18 & 1,26 & 13 & 4,81 & 6,154 & 4 \\
\hline K & 32 & 4 & 0,048 & 12 & 0,84 & 16 & 5,92 & 6,808 & 3 \\
\hline$S$ & 25 & 4 & 0,048 & 11 & 0,77 & 10 & 3,7 & 4,518 & 4 \\
\hline$P$ & 24 & 5 & 0,06 & 8 & 0,56 & 11 & 4,07 & 4,69 & 15 \\
\hline L & 23 & 4 & 0,048 & 7 & 0,49 & 12 & 4,44 & 4,978 & 2,5 \\
\hline $\mathrm{N}$ & 22 & 3 & 0,036 & 8 & 0,56 & 11 & 4,07 & 4,666 & 4 \\
\hline 0 & 20 & 6 & 0,072 & 7 & 0,49 & 7 & 2,59 & 3,152 & 3 \\
\hline & & 94 & 1,128 & 393 & 27,51 & 287 & 106 & 134,83 & \\
\hline
\end{tabular}

Fuente: Tesis "Evaluación De Las Reservas De Oro (Au) En Las Terrazas Eluviales Del Sector San Vicente" (Tsenkush F, 2016)

Tabla N² Colores y sitios de media concentración.

\begin{tabular}{|c|c|c|c|c|c|c|c|c|c|}
\hline \multicolumn{10}{|c|}{ SITIOS DE MEDIA CONCENTRACIÓN } \\
\hline \multicolumn{10}{|c|}{ COLORES } \\
\hline $\begin{array}{c}\text { Nombre } \\
\text { pozo }\end{array}$ & $\begin{array}{c}N^{\circ} \text { de } \\
\text { colores } \\
\text { m3 }\end{array}$ & $\begin{array}{c}\text { Malla } \\
\mathbf{6 0} \\
<0,3 \\
\text { mm } \\
\text { Trazas }\end{array}$ & $\begin{array}{c}\text { Peso } \\
\text { mg }\end{array}$ & $\begin{array}{c}\text { Malla } \\
\mathbf{3 5} \\
0,3-0,5 \\
\text { mm } \\
\text { Muy } \\
\text { Fino }\end{array}$ & $\begin{array}{l}\text { Peso } \\
\text { mg }\end{array}$ & $\begin{array}{c}\text { Malla } 18 \\
0,6-1 \\
\text { mm } \\
\text { Fino }\end{array}$ & $\begin{array}{c}\text { Peso } \\
\text { mg }\end{array}$ & $\begin{array}{l}\text { Peso } \\
\text { total }\end{array}$ & $\begin{array}{l}\text { Potencia } \\
\text { de } \\
\text { sobrecarga } \\
\text { (m) }\end{array}$ \\
\hline$M$ & 12 & 3 & 0,078 & 7 & 1,12 & 2 & 0,86 & 2,058 & 1,5 \\
\hline $\mathrm{H} 8$ & 11 & 2 & 0,052 & 7 & 1,12 & 2 & 0,86 & 2,032 & 2 \\
\hline
\end{tabular}




\begin{tabular}{cccccccccc}
$\mathrm{G}$ & 10 & 3 & 0,078 & 6 & 0,96 & 1 & 0,43 & 1,468 & 2 \\
$\mathrm{~V}$ & 14 & 2 & 0,052 & 11 & 1,76 & 1 & 0,43 & 2,242 & 3 \\
$\mathrm{Z}$ & 8 & 4 & 0,104 & 3 & 0,48 & 1 & 0,43 & 1,014 & 3 \\
$\mathrm{~F} 6$ & 8 & 3 & 0,078 & 4 & 0,64 & 1 & 0,43 & 1,148 & 3 \\
$\mathrm{I} 9$ & 6 & 2 & 0,052 & 3 & 0,48 & 1 & 0,43 & 0,962 & 2 \\
$\mathrm{D}$ & 5 & 2 & 0,052 & 3 & 0,48 & 0 & 0 & 0,532 & 2,5 \\
$\mathrm{U}$ & 5 & 1 & 0,026 & 4 & 0,64 & 0 & 0 & 0,666 & 1,5 \\
$\mathrm{I}$ & 3 & 1 & 0,026 & 2 & 0,32 & 1 & 0,43 & 0,776 & 3 \\
$\mathrm{~J}$ & 3 & 1 & 0,026 & 2 & 0,32 & 0 & 0 & 0,346 & 2 \\
$\mathrm{~J} 10$ & 4 & 1 & 0,026 & 3 & 0,48 & 0 & 0 & 0,506 & 1,5 \\
\hline & & 25 & 0,65 & 55 & 8,8 & 10 & 4,3 & 13,75 & \\
\hline
\end{tabular}

Fuente: Tesis "Evaluación De Las Reservas De Oro (Au) En Las Terrazas Eluviales Del Sector San Vicente" (Tsenkush F, 2016)

Tabla N³ Colores y sitios de baja concentración.

\section{SITIOS DE BAJA CONCENTRACIÓN}

\begin{tabular}{|c|c|c|c|c|c|c|c|c|c|}
\hline \multicolumn{10}{|c|}{ COLORES } \\
\hline $\begin{array}{c}\text { Nombre } \\
\text { pozo }\end{array}$ & $\begin{array}{c}\mathrm{N}^{\circ} \mathrm{de} \\
\text { colores } \\
\text { m3 }\end{array}$ & $\begin{array}{c}\text { Malla } \\
\mathbf{6 0} \\
<0,3 \\
\text { mm } \\
\text { Trazas }\end{array}$ & $\begin{array}{c}\text { Peso } \\
\text { mg }\end{array}$ & $\begin{array}{c}\text { Malla } \\
\mathbf{3 5} 0,3- \\
0,5 \mathrm{~mm} \\
\text { Muy } \\
\text { Fino }\end{array}$ & $\begin{array}{l}\text { Peso } \\
\text { mg }\end{array}$ & $\begin{array}{c}\text { Malla } \\
\mathbf{1 8} \\
0,6-1 \\
\text { mm } \\
\text { Fino }\end{array}$ & $\begin{array}{c}\text { Peso } \\
\text { mg }\end{array}$ & $\begin{array}{l}\text { Peso } \\
\text { total }\end{array}$ & $\begin{array}{l}\text { Potencia } \\
\text { de } \\
\text { sobrecarga } \\
(\mathrm{m})\end{array}$ \\
\hline G7 & 2 & 1 & 0,026 & 1 & 0,16 & 0 & 0 & 0,186 & 2 \\
\hline$E$ & 1 & 1 & 0,026 & 0 & 0 & 0 & 0 & 0,026 & 2,5 \\
\hline $\mathrm{F}$ & 2 & 0 & 0 & 2 & 0,32 & 0 & 0 & 0,32 & 2 \\
\hline $\mathrm{H}$ & 0 & 0 & 0 & 0 & 0 & 0 & 0 & 0 & 3 \\
\hline$\tilde{N}$ & 1 & 1 & 0,026 & 0 & 0 & 0 & 0 & 0,026 & 2 \\
\hline$T$ & 1 & 0 & 0 & 1 & 0,16 & 0 & 0 & 0,16 & 2 \\
\hline$x$ & 2 & 0 & 0 & 2 & 0,32 & 0 & 0 & 0,32 & 2,5 \\
\hline$Y$ & 0 & 0 & 0 & 0 & 0 & 0 & 0 & 0 & 2,6 \\
\hline & & 3 & 0,078 & 6 & 0,96 & 0 & 0 & 1,038 & \\
\hline
\end{tabular}

Fuente: Tesis "Evaluación De Las Reservas De Oro (Au) En Las Terrazas Eluviales Del Sector San Vicente" (Tsenkush F, 2016)

\section{Evaluación De Reservas Método Minero de Cortes o Perfiles.}

Consiste determinar la cantidad de mineral presente y la factibilidad de realizar la explotación comercial de dichos minerales metálicos mediante el trazado perfiles verticales del yacimiento y calcular las reservas de los bloques delimitados por dos perfiles. (García J. C., 2013)

Se usa cuando se tienen cuerpos mineralizados de desarrollo irregular y que han sido estudiados mediante afloramientos, excavaciones de calicatas y trincheras de forma que permiten establecer cortes o perfiles en los que se basa el cálculo de reservas. El área de 
la sección del cuerpo mineralizado interceptado por cada perfil se puede calcular por varios métodos (planímetro, regla de Simpson, etc.) (Cruz J., 2011).

Figura 5. Método Minero de Cortes o Perfil.

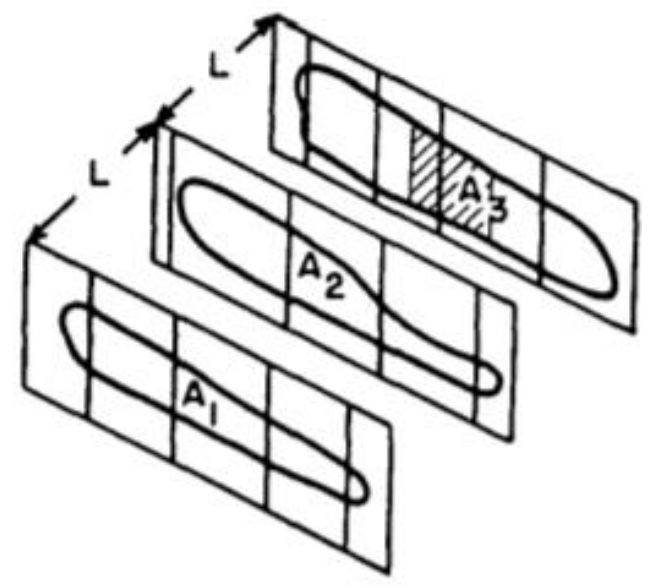

Fuente: Investigación de campo (Cruz, 2011).

Parámetro técnico para cálculo de tenores de oro $(\mathrm{Au})$.

$\mathrm{V}=$ Volumen del bloque entre dos secciones

$\mathrm{A} 1, \mathrm{~A} 2=$ Áreas de las secciones A1 y A2

$$
\mathrm{V}=\left(\frac{\mathrm{A} 1+\mathrm{A} 2}{2}\right) * \mathrm{~L} 1
$$

$\mathrm{T}=$ Peso en toneladas del bloque

$\mathrm{r}=$ Densidad media del mineral $\left(\mathrm{t} / \mathrm{m}^{3}\right)$

$$
\mathrm{T}=\left(\frac{\mathrm{A} 1+\mathrm{A} 2}{2}\right) * \mathrm{~L} 1 * \mathrm{~S}
$$

Método empleado para determinar oro (Au) $\mathbf{g r} / \mathbf{m}^{3}$ : se empleó concentración gravimétrica y conteo de chispas utilizando canalón y batea, A su vez se realizó una grilla de 9 secciones equidistantes, obteniendo los siguientes resultados:

- Alta concentración: Es aquella porción del recurso medido que en función a su alto porcentaje o nivel de concentración resulta interesante. El estrato mineralizado es $159.461,98 \mathrm{~m}^{3}$ con un tenor de $0.56 \mathrm{gr} / \mathrm{m}^{3}$ y reservas de 89.298 .70 gramos de oro. Tabla $\mathrm{N}^{\circ} 4$.

- Mediana concentración: es aquella porción del recurso indicado con discretos a medios niveles de concentración interesantes, bajo ciertos parámetros técnicos. El estrato mineralizado es $100486.86 \mathrm{~m}^{3}$ con un tenor de $0.085 \mathrm{gr} / \mathrm{m}^{3}$ y reservas de 8541.38 gramos de oro. Ver la Tabla $\mathrm{N}^{\circ} 5$.

- Baja concentración: Es aquella porción de recurso que contiene escasa concentración, su interés está en función a la cantidad del recurso. El estrato mineralizado es de $29657,25 \mathrm{~m}^{3}$ con un tenor de $0.016 \mathrm{gr} / \mathrm{m}^{3}$ y reservas de 474.51 gramos de oro. Tabla $\mathrm{N}^{\circ} 6$. 
Tabla N 4 Reservas de Alta Media y Baja ley.

\begin{tabular}{ccccc}
\hline \multicolumn{5}{c}{ Reservas de Alta Media y Baja ley } \\
\hline PERFIL & Distancia & Volumen & $\begin{array}{l}\text { Tenor } \\
\text { gr/m }\end{array}$ & $\begin{array}{c}\text { Reservas de } \\
\text { oro (gr) }\end{array}$ \\
\hline$(\mathrm{A} 1+\mathrm{A} 2) \mathrm{d} /$ & $150 / 2$ & 27649.50 & & \\
\hline$(\mathrm{A} 2+\mathrm{A} 5) \mathrm{d} /$ & $150 / 2$ & 30265.12 & \\
\hline$(\mathrm{A} 5+\mathrm{A} 6) \mathrm{d} /$ & $150 / 2$ & 28010.62 & \\
\hline$(\mathrm{A} 6+\mathrm{A} 7) \mathrm{d} /$ & $150 / 2$ & 32651.25 & \\
\hline$(\mathrm{A} 7+\mathrm{A} 8) \mathrm{d} /$ & $150 / 2$ & 23392.12 & \\
\hline$(\mathrm{A} 8+\mathrm{A} 9) \mathrm{d} /$ & $150 / 2$ & 17493.37 & \\
\hline & & 159461.98 & 0.56 & 89298.70 \\
\hline
\end{tabular}

Fuente: Tesis "Evaluación De Las Reservas De Oro (Au) En Las Terrazas Eluviales Del Sector San Vicente" (Tsenkush F, 2016)

Tabla № 5 Reservas de mediana concentración.

\begin{tabular}{ccccc}
\hline \multicolumn{5}{c}{ Zonas con reservas de mediana concentración de oro } \\
\hline Perfiles & $\begin{array}{c}\text { Distancia } \\
(\mathbf{m})\end{array}$ & Volumen $\left(\mathbf{m}^{3}\right)$ & $\begin{array}{c}\text { Tenor } \\
\left(\mathbf{g r} / \mathbf{m}^{3}\right)\end{array}$ & $\begin{array}{c}\text { Reservas } \\
\mathbf{d e} \text { oro } \\
(\mathbf{g r})\end{array}$ \\
\hline$(\mathrm{A} 2+\mathrm{A} 3) \mathrm{d} / 2$ & $150 / 2$ & 5250 & \\
\hline$(\mathrm{A} 3+\mathrm{A} 4) \mathrm{d} / 2$ & $150 / 2$ & 12342.37 & \\
\hline$(\mathrm{A} 4+\mathrm{A} 5) \mathrm{d} / 2$ & $150 / 2$ & 22758.75 & \\
\hline$(\mathrm{A} 5+\mathrm{A} 6) \mathrm{d} / 2$ & $150 / 2$ & 19614.37 & \\
\hline$(\mathrm{A} 6+\mathrm{A} 7) \mathrm{d} / 2$ & $150 / 2$ & 22944.37 & \\
\hline$(\mathrm{A} 7+\mathrm{A} 8) \mathrm{d} / 2$ & $150 / 2$ & 17577 & \\
\hline
\end{tabular}

Fuente: Tesis "Evaluación De Las Reservas De Oro (Au) En Las Terrazas Eluviales Del Sector San Vicente" (Tsenkush F, 2016)

Tabla N 6 Reservas de baja concentración de Oro.

\begin{tabular}{ccccc}
\hline \multicolumn{5}{c}{ Zonas con reservas de baja concentración de oro } \\
\hline PERFIL & $\begin{array}{l}\text { Distancia } \\
(\mathbf{m})\end{array}$ & Volumen $\left(\mathbf{m}^{3}\right)$ & $\begin{array}{l}\text { Tenor } \\
\mathbf{g r} / \mathbf{m}^{3}\end{array}$ & $\begin{array}{l}\text { Reservas } \\
\text { de oro } \\
(\mathbf{g r})\end{array}$ \\
\hline$(\mathrm{A} 4+\mathrm{A} 5) \mathrm{d} / 2$ & $150 / 2$ & 16933.12 & \\
\hline
\end{tabular}




\begin{tabular}{cccc}
$(\mathrm{A} 5+\mathrm{A} 6) \mathrm{d} / 2$ & $150 / 2$ & 12724.12 & \\
& & & \\
\hline & 29657.2 & 0.016 & 474.51 \\
\hline
\end{tabular}

Fuente: Tesis "Evaluación De Las Reservas De Oro (Au) En Las Terrazas Eluviales Del Sector San Vicente" (Tsenkush F, 2016)

Tabla $\mathbf{N}^{\circ} 7$ Cálculo matemático en sitios de alta, mediana y baja ley de oro $(\mathrm{Au})$.

\begin{tabular}{ccccc}
\hline \multicolumn{5}{c}{ Sitios de alta, mediana y baja ley de oro (Au). } \\
\hline $\begin{array}{c}\text { Sitios de áreas } \\
\text { probadas }\end{array}$ & $\begin{array}{c}\Sigma \text { de secciones } \\
\text { (cuerpo } \\
\text { mineralizado) } \mathrm{m}^{3}\end{array}$ & $\begin{array}{c}\text { Tenor del depósito } \\
\mathrm{gr} / \mathrm{m}^{3}\end{array}$ & $\begin{array}{c}\text { Reservas } \\
\text { del depósito } \\
\text { gr de Au }\end{array}$ & $\begin{array}{c}\text { Sobrecarga } \\
\text { total } \mathrm{m}^{3}\end{array}$ \\
\hline $\begin{array}{c}\text { Alta } \\
\text { Concentración } \\
\text { Mediana } \\
\text { concentración }\end{array}$ & $159.461,98 \mathrm{~m}^{3}$ & $0.56 \mathrm{gr} / \mathrm{m}^{3}$ & $89.298,70$ & \\
$\begin{array}{c}\text { Baja } \\
\text { concentración }\end{array}$ & $29.657,25 \mathrm{~m}^{3}$ & $0.016 \mathrm{gr} / \mathrm{m}^{3}$ & 474.51 & \\
\hline & $254846,34 \mathrm{~m}^{3}$ & Total reservas: $98314,59 \mathrm{gr}$ de Au. & $365104,52 \mathrm{~m}^{3}$ \\
\hline
\end{tabular}

Fuente: Tesis "Evaluación De Las Reservas De Oro (Au) En Las Terrazas Eluviales Del Sector San Vicente" (Tsenkush F, 2016)

\section{Sistema y método de explotación propuesto}

Características técnicas para la elección del método de explotación. Analizando en la zona de estudio la geología local, la disposición del depósito, la topografía, la hidrogeología de la zona, se establece que se puede realizar extracción de este depósito tanto por el método seco mediante el uso de excavadoras como por el método húmedo mediante el uso de motobombas y bombas de succión; consecuente también por métodos mixtos o combinados. (Cisneros, 2003). Ver la Tabla $\mathrm{N}^{\circ} 8$ donde se reflejan los costos y características técnicas de cada equipo. (Vasquez E., 2016)

Tabla № 8 Características técnicas para la elección del método de explotación.

\begin{tabular}{cccccr}
\hline $\begin{array}{c}\text { Equipos de } \\
\text { trabajo }\end{array}$ & $\begin{array}{c}\text { Alquiler de } \\
\text { maquinaria }\end{array}$ & Combustible & Tubos & $\begin{array}{c}\text { Número de } \\
\text { Personal }\end{array}$ & Alimentación \\
\hline $\begin{array}{c}\text { 1 Motobomba } \\
\text { de 3" }\end{array}$ & $\begin{array}{c}40,00 \text { USD } \\
\text { la hora } 12 \\
\text { meses }\end{array}$ & $\begin{array}{c}92.928 \\
\text { galones por } \\
\text { año X } 1,48 \\
\text { USD/gal }\end{array}$ & $\begin{array}{c}300 \mathrm{~m} \text {. de } \\
\text { manguera } \\
\text { de } 3 \text { inch }\end{array}$ & $\begin{array}{c}3 \text { personas } \\
\text { laboran todo } \\
\text { el año }\end{array}$ & \\
$\begin{array}{c}\text { 1concentradora Z } \\
\text { tipo } \text { canalón } \\
6.950,00\end{array}$ & $15.360,00$ & $12.503,04$ & 525 & $15.840,00$ & $2.376,00$ \\
\hline $\begin{array}{c}\text { Maquinaria y } \\
\text { equipos de } \\
\text { trabajo }\end{array}$ & $\begin{array}{c}\text { Costo por } \\
\text { hora de } \\
\text { trabajo } \\
\text { efectivo }\end{array}$ & Combustible & Tubos & $\begin{array}{c}\text { Número de } \\
\text { Personal }\end{array}$ & Alimentación \\
\hline
\end{tabular}




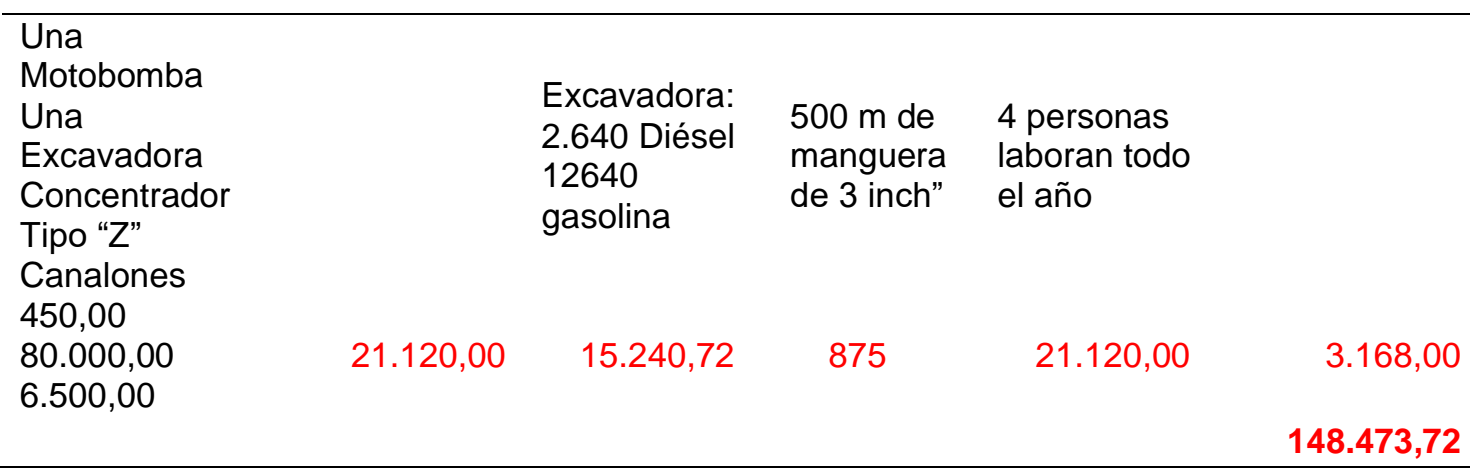

Fuente: Maquinaria. Andrade, E. (2012).

Diseño del método de explotación por franjas y arranque por medios hidráulicos, sirviéndose de los afloramientos del yacimiento descubiertos por la bisección de la quebrada Kayamas al yacimiento; y conforme a la experiencia de campo, el arranque hidráulico es de menor coste, pero exige de mayor control ambiental, ya que genera turbiedad, sedimentos, sólidos en suspensión, en fuentes de agua, así como el cambio geomorfológico (Granizo F., 2016).

Diseño del método de explotación por franjas y arranque por medios mecánicos, este método técnicamente se ajusta al yacimiento en estudio por las características geológicas, litológicas y geotécnicas; adicionalmente cabe destacar que el yacimiento tiene sobre carga de regolita que fluctúa de 15 a $30 \mathrm{~m}$ de potencia; a este método se le considera como un sistema de mayor coste en la producción minera; de igual manera se debe considerar la legislación minera vigente que limita considerablemente las capacidades de maquinaria permitidas en la modalidad de minería artesanal (Granizo F., 2016).

En base al volumen y la calidad del estrato mineralizado, y de sobrecarga, se realiza el análisis del método de explotación, considerando maquinaria y equipo tanto en frente de avance por franjas y arranque por medios hidráulicos como en frente de avance por franjas y arranque por medios mecánicos; los costos de implementación, operación y producción que implicaría para el desarrollo de explotación. Estimación de costos de inversión y capital de recuperación.

La TIR del 49.74\%, comparada con la TMAR del $12.06 \%$ es mucho mayor, lo que implica que el proyecto en el tiempo es aceptable, porque va a rendir un $37.68 \%$, VAN $(\$ 60464,35)$ adicionales.

En el frente de avance por franjas y arranque por medios mecánicos: La TIR del 3.39\%, comparada con la TMAR del $12.06 \%$ es mucho menor, siendo el valor negativo de VAN (\$-31809.96) lo que implica que el proyecto no recuperara ni lo mínimo esperado, de la forma de explotación que en la actualidad aplican los mineros artesanales. Ver datos en la Tabla $\mathrm{N}^{\circ} 9$. 
Tabla N9 Factibilidad Económica.

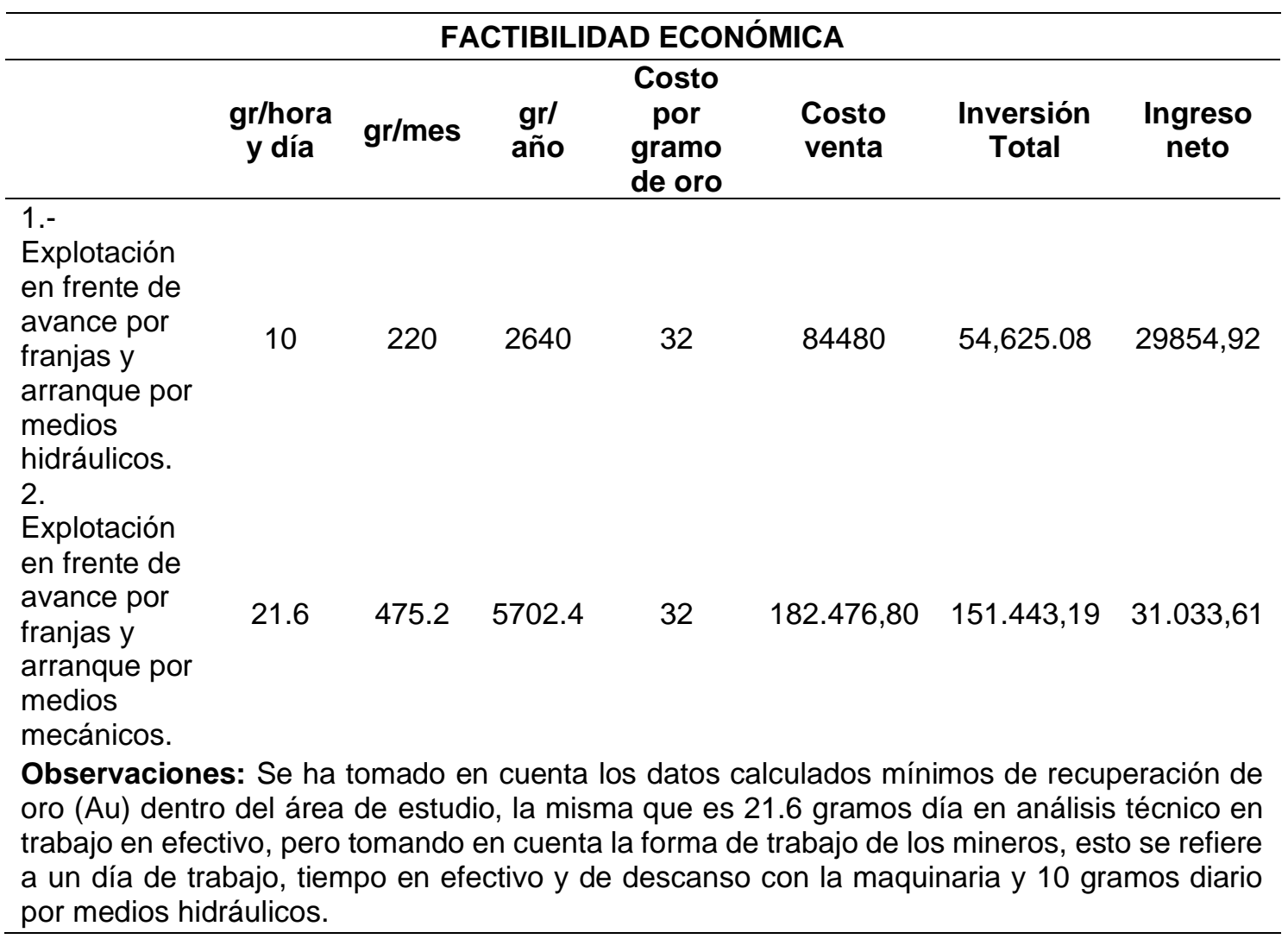

Fuente: Tesis "Evaluación De Las Reservas De Oro (Au) En Las Terrazas Eluviales Del Sector San Vicente" (Tsenkush F, 2016)

\section{Discusión}

La comunidad Shuar de San Vicente donde se asientan permisos de minería artesanal objetivos de la investigación dispuso ciertas restricciones al acceso en su territorio, como el reducido ingreso de personal, el trabajo de investigación exclusivamente en el campo y el impedimento del uso de maquinaria. Por estas restricciones las muestras de arena aurífera no fueron analizadas en un laboratorio, el muestreo se hizo manualmente y la metodología de muestreo en el campo fue mediante líneas transversales (método de zonas) a los ejes de las quebradas: Yawaimi, Kayamas, Tsuntsuntsa con limpiezas de afloramientos y ejecución de calicatas en los márgenes de las quebradas antes mencionadas; se evitó muestrear puntos altos de la topografía, siendo una ventaja de este método el obtener información de manera rápida sin un en mallado que implicaría mayor intervención en la vegetación que cubre la zona, los datos recolectados nos sirvieron para aplicar la metodología de estimación por cálculo de secciones, lo cual no aporta con datos sobreestimados pero si con un factor de subestimación manejable; al no poder utilizar laboratorio se recurrió al conteo de chispas mediante métodos gravimétricos y balanza digital de campo en presencia de miembros de la comunidad.

Bajo las condiciones que se pudo trabajar en la investigación de esta zona, el método antes descrito aporta de valores fiables pero sin embargo de alguna manera si hubiese 
mayor apertura de la comunidad se podría haber realizado un muestreo mediante un mallado más denso, con aplicación de maquinaria en aperturas de calicatas de mayor profundidad y con el análisis en laboratorio se hubiese obtenido datos más certeros para la aplicación de la geo estadística para su mejor tabulación, como las pruebas de oro total y su calidad de recuperación, al haber aplicado este primer método es importante considerar un estudio a mayor detalle.

Al planificar la investigación de campo y en base de datos preliminares se llegó a la conjetura de encontrar una terraza homogénea, de manera particular en los lechos y márgenes de las quebradas, pero en el muestreo se obtuvo zonas de concentraciones de alta, media y baja ley. En las zonas de alta ley como de mediana ley revelan la mayor acumulación chispas en el orden muy finas, 0,3-0,5 mm, seguida en fino 0,6-1 $\mathrm{mm}$ y en menor proporción en trazas $<0,3 \mathrm{~mm}$. Cabe señalar en referencia a la granulometría de la graba esta predominantemente constituida por arena de material silíceo de origen marino (fósiles) y la cobertura de sobrecarga está constituido por lutitas, estableciéndose que el yacimiento aurífero no recibió aporte alguno de la cordillera ya que ningún material de esta interviene en la constitución de este y la deposición de mayor número de chispas está en el orden predominante de muy finas. Por esta razón este placer aurífero no aporta con datos para la exploración de primario en la zona. Los sitios de mayor concentración en reservas de 89.298.70 gramos de oro, nos indica que sigue la disposición característica de la mayoría de los yacimientos de la zona de poca extensión con leyes medias a altas.

La decisión que se determinó para un estudio de factibilidad con el análisis del método tradicional mediante arranque hidráulico con hidro monitores artesanales y el propuesto mediante destape de la sobre carga con maquinaria y el arranque hidráulico fue claramente comparativa en función de costos sin considerar obras complementarias, con el fin de visualizar su rentabilidad con los actuales datos recopilados.

Este trabajo esta categorizado dentro de prospección y exploración inicial ya sea por el método de muestreo de líneas transversales el método de estimación de reservas cálculo de secciones a diferencia del estudio Exploración y Explotación de Terrazas Aluviales en la concesión "El Icho" llevado a cabo por Mendoza Salas Patricia Jaqueline (2015) "Se inicia la exploración avanzada, mediante la elaboración de pozos, distribuidos en una malla de $25 \mathrm{~m}$ con la utilización de una excavadora, con un cucharon de volumen 0.85 $\mathrm{m}^{3}$ y una mini planta de recuperación tipo Trommel como equipos principales, en el sector" (Mendoza P., 2015)

Esta investigación se realizó con distancias entre líneas de 150 m, muestreo manual y mediante observación in situ mediante conteo de chispas. También, la topografía del sector en este sitio va desde un relieve tipo montañoso (estribaciones de cordillera del Cutucú) a una llanura sin un desnivel muy marcado, a diferencia de otras terrazas del sector que comúnmente como se describe "las llanuras aluviales que se ubican entre las colinas anteriores. Existen un sinnúmero de pantanos, zonas y depresiones inundadas, regadas a lo largo de los actuales cauces de los ríos “. (Mendoza P., 2015). 
Se ha obtenido 38 muestras positivas recolectadas, razón por la cual se utilizó la estimación de cálculo por perfiles en cambio en exploraciones avanzadas de pequeña minera como indica textualmente al haber gran cantidad de datos "El método de cubicación empleado para este cálculo es el de Área de Influencia, media Aritmética". (Mendoza P., 2015).

En comparación al estudio Diseño de Explotación de los Depósitos Auríferos Aluviales Profundos del Río Jatun Yacu en la cual indica referente al sistema de explotación en relación al génesis del yacimiento y sus características geométricas "Por la génesis del yacimiento y sus características geométricas y técnico mineras, el método de explotación establecido para este tipo de depósito es el "Método de explotación No Selectivo a cielo abierto, en frente continuo de corte y relleno del espacio explotado en franjas longitudinales al curso del río.", en nuestro caso no es posible ese sistema de explotación, primero por su tamaño del yacimiento, topografía y el sistema hídrico, también por la calificación minera ya que este es minería artesanal, razón por la cual no es permitido industrialización en su explotación por ser minería de subsistencia, de tal manera se plantea la explotación hidráulica mediante hidro monitores , o una mixta maquinaria para la limpieza de sobre carga y el arranque mediante hidromonitores.

\section{Conclusiones.}

- En la terraza aluvial se establece el cálculo de volumen por el método de perfiles de estratos mineralizados de alta concentración en 159.461,98 $\mathrm{m}^{3}$ con un tenor de $0.56 \mathrm{gr} / \mathrm{m}^{3}$ y reservas de $89.298,70$ gramos de oro, Mediana concentración $100.486,86 \mathrm{~m}^{3}$ con un tenor de $0.085 \mathrm{gr} / \mathrm{m}^{3}$ y reservas de 8.541 .38 gramos de oro, Baja concentración $29.657 .25 \mathrm{~m}^{3}$ con un tenor de $0.016 \mathrm{gr} / \mathrm{m}^{3}$ y reservas de 474.51 gramos de oro., obteniéndose unas reservas probadas totales de 98.314,59 gramos de oro.

- Según el levantamiento geológico de campo realizado, se determinó que en la zona de estudio existen zonas de hundimiento en las cuales no existen estratos mineralizados.

- Las actividades mineras actuales aprovechan el material por desgaste del pie de talud y paralizan la actividad cuando han liquidado la capa aurífera, dejando inestable los taludes a la espera que la quebrada crezca y remueva la sobrecarga.

- El arranque por franjas y arranque por medios hidráulicos es de menor coste de operación que el de franjas y arranque por medios mecánicos, pero con mayor inversión de obras de mitigación y control ambiental.

- El diseño de Explotación de arranque por medios mecánicos, no se puede aplicar por la restricción de capacidades técnicas de la maquinaria en la ley de minería.

- Se trabaja en tres grupos de colores: Malla $60<0,3$ mm Trazas; Malla 35 0,3-0,5 mm Muy Fino; Malla 18 0,6-1 mm Fino. 


\section{Referencias bibliográficas.}

Andrade, E. (21 de mayo de 2012). Motobombas con motor a gasolina de 3". (T. Ltda, Editor, \& Barranquilla, Colombia) Recuperado el 12 de septiembre de 2015, de http://www.equinorte.net/index.php/2012-05-21-00-11-18/2012-05-21-01-24-

38/maquinaria-para-bombeo-aguas-limpias

Baldock W, J. (1982). Geología del Ecuador. Quito: Dirección General de Geología y Minas.

Cisneros, J. (octubre de 2003). Diseño de explotación a cielo abierto. (Sistemas y métodos de explotación) Recuperado el 28 de octubre de 2015, de http://www.aimecuador.org

Cruz, J. (29 de junio de 2011). Cálculo de Reservas. (Métodos Clásicos o Geométricos) Recuperado el10 de febrero de 2016, de http://es.scribd.com/doc/58981418/Cálculode-reservas\#scribd

García J. C. (02 de diciembre de 2013). Evaluación de los yacimientos minerales. (Prezi, Editor, \& México) Recuperado el 15 de septiembre de 2016, de Evaluación de reservas: https://prezi.com/x4kebe5zhkax/evaluación-de-los-yacimientos-minerales/

Granizo, F. (2016). Diseños de explotación. (F. A. Tsenkush, Entrevistador) Asesoría \& Consultoría de sectores estratégicos. Quito, Ecuador. 20 de Febrero 2016.

Mendoza P. (2015). Exploración y explotación de terrazas aluviales en la concesión "El Icho". Trabajo de grado para obtención de título de Ingeniero en Geología y Minas. Escuela Superior Politécnica de Chimborazo. Riobamba

Swiecki, R. (2008). Exploación y minería alluvial. (Aluviales y eluviales depósitos de oro o diamantes) Tomado del siguiente enlace: www.minelinks.com/alluvial/deposits_es.html

Tsenkush F., (2016). Evaluación de las reservas de Oro (Au) en las Terrazas eluviales del Sector San Vicente". Trabajo de grado para obtención de título de Ingeniero en Geología y Minas. Escuela Superior Politécnica de Chimborazo. Riobamba, Ecuador.

Vásquez, E. (2016). Manejo de estériles de una mina. Maracaibo.

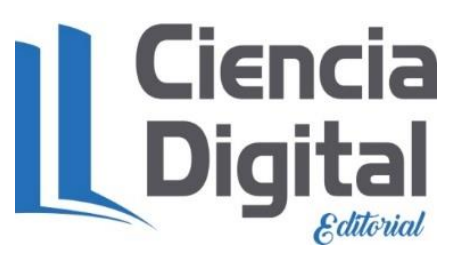




\section{PARA CITAR EL ARTÍCULO INDEXADO.}

Mejía Flores, M. A., Granja Carrera, J. P., \& Tsenkush Chamik, F. A. (2021). Evaluación de las reservas de oro $(\mathrm{Au})$ en las terrazas aluviales del sector San Vicente. ConcienciaDigital, 4(3), 94-110. https://doi.org/10.33262/concienciadigital.v4i3.1766

\section{Liencia}

El artículo que se publica es de exclusiva responsabilidad de los autores y no necesariamente reflejan el pensamiento de la Revista Conciencia Digital.

El artículo queda en propiedad de la revista y, por tanto, su publicación parcial y/o total en otro medio tiene que ser autorizado por el director de la Revista Conciencia Digital.

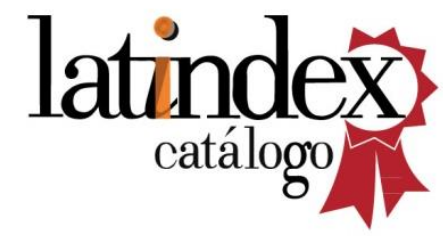

